\title{
Aspectos nutricionais associados ao envelhecimento de indivíduos com síndrome de Down: uma revisão integrativa
}

\author{
Andreia Araujo Lima Torres*
}

\section{Resumo}

Este trabalho apresenta uma análise da literatura acerca das pesquisas mais recentes envolvendo a alimentação e suplementação durante o envelhecimento de indivíduos com síndrome de Down. Em decorrência da alta incidência de Alzheimer, torna-se relevante o estudo de fatores que possam contribuir para uma melhor qualidade de vida nesta população. Os custos financeiros, emocionais e familiares na doença de Alzheimer são grandes e aumentam na ausência de uma terapêutica que retarde a progressão dessa. Dessa forma, foi realizada uma busca bibliográfica, referentes aos últimos 10 anos, nas bases de dados Scielo e Bireme. O objetivo foi levantar estudos que relacionassem a alimentação e a suplementação de compostos específicos ao processo de envelhecimento em indivíduos com SD. A análise dos documentos extraídos levou à leitura de 38 artigos. Parte das pesquisas na área de nutrição focaram na importância da neutralização dos radicais livres e na redução da inflamação cerebral como estratégia remediativa para as complicações mais comuns encontradas nessa população. Outros aspectos do envelhecimento de indivíduos com síndrome de Down foram abordados.

Palavras-chave: Síndrome de Down. Doença de Alzheimer. Suplementação alimentar.

\section{Introdução}

A síndrome de Down (SD) é uma condição genética resultante da trissomia do cromossomo 21. Estima-se que 1 em cada 700-750 nascidos vivos sejam diagnosticados com a síndrome (PARKER et al., 2010). Ao invés de 46 cromossomos (23 herdados da mãe e 23 do pai), o indivíduo com SD possui uma trissomia livre. Assim, no total, são 47 cromossomos em todas ou na maior parte das células. Características comuns dessa condição incluem baixa estatura, hipotonia muscular e deficiência intelectual (KIRWAN et al., 2015; STAGNI et al., 2015).

Também é observada maior incidência de doenças cardiovasculares, diabetes, leucemia, hipotireoidismo, obesidade, constipação intestinal, apneia respiratória (STRUPP et al., 2016).

Observa-se neste grupo um envelhecimento mais acelerado, acompanhado de aparecimento precoce de rugas, cabelos brancos, hipogonadismo, menopausa, declínio da função imune (ZIGMAN,

* Nutricionista, mestre em nutrição humana, doutora em psicologia clínica e cultura, pós-doutoranda e pesquisadora em Saúde Coletiva, Universidade de Brasília (UnB). E-mail: andreiat@me.com Endereço para Correspondência: Faculdade de Ciências da Saúde - Departamento de Saúde Coletiva. Programa de Pós-Graduação em Saúde Coletiva, Campus Universitário Darcy Ribeiro - Asa Norte - CEP 70901-900 Brasília, DF.

$\rightarrow$ http://dx.doi.org/10.5335/rbceh.v13i2.5798

Recebido em: 09.03.2016. Aceito em: 09.11.2016. 
2013) e demência precoce associada ao mal de Alzheimer (THIEL; FOWKES, 2007).

Destacam-se como fatores etiológicos para a SD a idade materna avançada (WU; MORRIS, 2013a), a presença de polimorfismos (MATHUSWAMY; AGARWAL, 2016) e dificuldades na metilação do DNA (BACALINI et al., 2015; COPPEDÈ et al., 2013). A metilação do DNA refere-se a alterações epigenéticas que provocam o silenciamento de determinados genes (SILVA et al., 2010). No caso da SD, como há um cromossomo sobressalente, a metilação torna-se importante para que a superexpressão de determinados genes seja inibida. Falhas no processo aumentam o risco de determinadas doenças, dentre elas o Alzheimer (LU et al., 2016; MENTIS, 2016).

Alguns substratos são fundamentais para os processos de metilação, como o aminoácido metionina e a colina, que atuam como substratos em vias de metilação, além de cofatores vitamínicos (B2, B6, B9 e B12). Em decorrência da indispensabilidade de tais nutrientes para os processos de metilação, pesquisadores da área destacam que alimentação inadequada na gestação, gera carências que aumentam o risco de mudanças epigenéticas sistemáticas na prole (DELCURTO; WU; SATTERFIELD, 2013; DOMINGUEZ-SALAS et al., 2014; WESSELS, 2014).

Tais conhecimentos e os recentes avanços da medicina permitiram um aumento da esperança média de vida no grupo de pessoas com SD. Contudo, esta ainda é menor do que à do restante da população, justificando o estudo de estratégias que possam garantir o adequado desenvolvimento e a saúde desses por toda a vida (WU; MORRIS, 2013b). Apesar de muito se escrever no Brasil sobre o envelhecimento de indivíduos na síndrome de Down, poucos autores dedicam-se ao tema específico da alimentação e suplementação. $\mathrm{O}$ banco de Teses e Dissertações da Capes, por exemplo, identifica 12 documentos referentes a trabalhos concluídos sobre alimentação e nutrição na SD, entre 2013 e 2016. Desses, 9 foram realizados com crianças e/ou adolescentes e 3 com adultos, sendo o foco principal o estudo sobre a imunidade de indivíduos com SD. Outras temáticas envolveram a avaliação do estado nutricional, a saúde bucal e as disfunções tireoidianas.

Dessa forma, o objetivo desta revisão integrativa é levantar estudos que relacionem a alimentação e a suplementação de compostos específicos ao processo de envelhecimento em indivíduos com SD. A hipótese é a de que tais compostos podem contribuir para uma melhor qualidade de vida durante o envelhecimento e para o retardo do Alzheimer na SD.

\section{Metodologia}

Foi realizada uma revisão integrativa dos últimos 10 anos (janeiro de 2006 a fevereiro 2016), por meio de análise de publicações científicas contemplando o tema síndrome de Down e envelhecimento. Foram consultadas as bases de dados Scielo e Bireme. A busca foi realizada no mês de fevereiro de 2016. As palavras- 
-chave utilizadas foram: Síndrome de Down, Envelhecimento, Alimentação, Nutrição e Suplementação, nas línguas português e inglês. Critérios de inclusão incluíram ser artigo científico com disponibilidade do texto na íntegra, publicação em periódico revisado por pares, ano de publicação (2006 a 2016). Todos os tipos de pesquisa foram consideradas (revisão, estudos experimentais e estudos de caso). Foram excluídos artigos duplicados, que não foram publicados nas línguas português ou inglês, que apresentaram pesquisa fora do escopo da busca, que não foram publicados em revista científica indexada e avaliada por pares ou que trataram da síndrome de Down na infância ou adolescência.

A busca nas bases de dados gerou 432 documentos. Inicialmente, 43 documentos duplicados e 10 textos sem acesso ao material na íntegra foram descartados. Foram excluídos ainda 74 outros documentos, sendo: 32 monografias, teses e dissertações, 7 projetos pedagógicos de cursos, 2 provas de concurso, 16 anais de congressos e 17 livros. Após esta fase, foi feita a leitura dos títulos e resumos dos artigos restantes $(\mathrm{n}=305)$. Tal leitura possibilitou a exclusão de outros 209 artigos não relacionados diretamente ao tema ou à área da pesquisa (Tabela 1).

Tabela 1 - Artigos excluídos após leitura de títulos e resumos

\begin{tabular}{l|c|l|c}
\hline \multicolumn{1}{c|}{ Área ou tema do artigo } & Descartados & \multicolumn{1}{|c}{ Área ou tema do artigo } & Descartados \\
\hline Atenção primária & 5 & Crescimento e desenvolvimento infantil & 18 \\
Cuidados enfermagem & 10 & Diabetes & 7 \\
Direitos do deficiente & 11 & Educação Física & 19 \\
Farmácia & 4 & Fisioterapia & 15 \\
Fonoaudiologia & 3 & Gestação e lactação & 6 \\
Idosos & 4 & Medicina veterinária & 2 \\
Medicina (diferentes áreas) & 27 & Métodos de pesquisa & 3 \\
Nutrição (outros aspectos) & 13 & Obesidade & 4 \\
Odontologia & 15 & Outros assuntos & 21 \\
Paralisia cerebral & 3 & Psicologia & 3 \\
Qualidade de vida dos cuidadores & 9 & Sexualidade & 2 \\
Teologia & 2 & Terapia Ocupacional & 4 \\
Transtorno do espectro do autismo & 3 & Total de artigos descartados: & 209 \\
\hline
\end{tabular}

Fonte: dados da pesquisa.

Após a leitura completa dos 96 artigos restantes, 37 foram utilizados na revisão por apresentarem objetivos, método e resultados bem definidos e condizentes com o objeto da pesquisa (Figura 1). 
Figura 1 - Fluxograma do processo de seleção dos artigos pesquisados

Exclusão inicial de 53 documentos (formato distinto de artigo científico, impossibilidade de acesso ao documento na íntegra ou duplicação). Exclusão de outros 209 artigos sem relação direta ao tema da pesquisa.

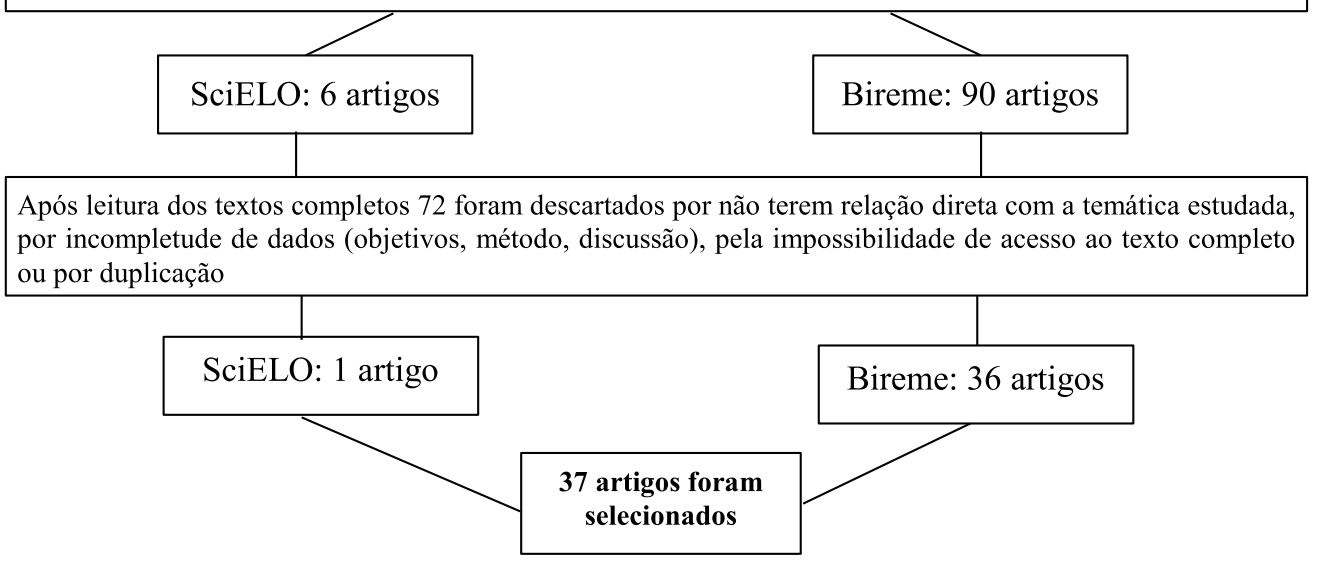

Fonte: dados da pesquisa.

Toda a avaliação foi feita por um único pesquisador. A Tabela 2 traz os resultados finais, referente aos 37 ar-

Tabela 2 - Resultados da busca sist

\section{tigos selecionados a partir de termos específicos utilizados na busca.}

\begin{tabular}{l|c|c|c|c|c|c}
\hline \multicolumn{1}{c|}{ Base de dados } & \multicolumn{2}{c|}{ Scielo } & \multicolumn{3}{c}{ Bireme } \\
\hline \multicolumn{1}{c|}{ Termos } & Encontrados & Exclusão & Total & Encontrados & Exclusão & Total \\
\hline Síndrome de Down Envelhecimento / Down & 1 & 1 & 0 & 67 & 37 & 30 \\
Syndrome AND Aging & & & & & \\
Síndrome de Down AND Alimentação & 0 & 0 & 0 & 0 & 0 & 0 \\
Down Syndrome AND eating & 1 & 1 & 0 & 3 & 2 & 1 \\
Síndrome de Down AND Nutrição & 3 & 3 & 0 & 4 & 1 & 3 \\
Down Syndrome AND Nutrition & 0 & 0 & 0 & 6 & 6 & 0 \\
Síndrome de Down AND Suplementação & 1 & 0 & 1 & 2 & 2 & 0 \\
Dow Syndrome AND Supplementation & 0 & 0 & 0 & 8 & 6 & 2 \\
$\quad$ Sub total & 6 & 5 & $\mathbf{1}$ & 90 & 53 & $\mathbf{3 6}$ \\
$\quad$ Total & & & & & $\mathbf{3 7}$ \\
\hline
\end{tabular}

Fonte: dados da pesquisa. 
Resultados

A maior parte dos estudos selecionados foi publicado em inglês $(n=34$, $92 \%$ ) e apenas 2 em português (8\%). Dentre os 37 artigos que fazem parte da presente revisão, $22(59,4 \%)$ são estudos experimentais e $15(40,6 \%)$ são revisões de literatura. Os dois artigos publicados em português fazem parte desta última categoria de pesquisa.

Grande parte dos trabalhos selecionados $(\mathrm{n}=11,28 \%)$ abordaram aspectos relacionados ao Alzheimer, a maior preocupação relatada pelos pesquisadores durante o envelhecimento de indivíduos com Síndrome de Down. Outros assuntos abordados incluíram a disfunção mitocondrial $(\mathrm{n}=6,16 \%)$, o estresse oxidativo ( $\mathrm{n}=6,16 \%)$, o uso de compostos fenólicos ( $\mathrm{n}=4,10 \%)$ e vitaminas como alternativa terapêutica no envelhecimento (n $=4,10 \%)$.

Os estudos selecionados e seus principais resultados são apresentados na Tabela 3.

Tabela 3 - Artigos selecionados para a revisão a partir das bases de dados Scielo e Bireme, sobre o tema "Aspectos nutricionais relacionados ao envelhecimento na Síndrome de Down"

Continua

\begin{tabular}{|c|c|c|c|c|}
\hline Aulnt, คпо & Linguя & Método & Objetivo & Felevïncія рагя о presenle arligo \\
\hline BACALINI a: al., 2015. & Inglès & Experimen:s & $\begin{array}{l}\text { Investigar os padrōes ce metilaç̃o co DVA cm } \\
\text { amostas ce sangue ce pessous com sindrome } \\
\text { ce Down c en seus parentes sem SD. }\end{array}$ & 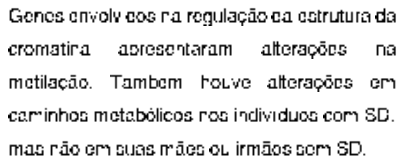 \\
\hline B AĞl c: al.. 2014. & Ingläs & Experimen:s & $\begin{array}{l}\text { Analisar a composiçāo ca mic robiota ce pes- } \\
\text { soas com SD o sua re ação com o cn̛elhec- } \\
\text { mento. }\end{array}$ & 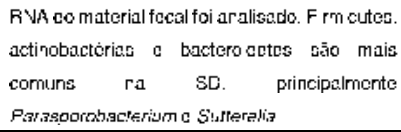 \\
\hline $\begin{array}{l}\text { BUT-EAFIEL } \supset \text { a: al., } \\
201 \div .\end{array}$ & Inglès & Revisāo & $\begin{array}{l}\text { D scuir as cyidenc as ace'ca cos mecanismos } \\
\text { cLe ligam o cot resse oxida: vo ae cesenvolyi- } \\
\text { merto ca ceméncia ra SD. }\end{array}$ & 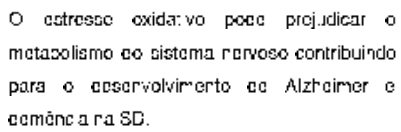 \\
\hline CENINI ct al., 2015. & Inglès & Experimen:s & $\begin{array}{l}\text { Irves:igar a re aç̄o crtre a idade a SD c a neu- } \\
\text { ropatia ca cocręa ce Alzłcimer ros rive s ce } \\
\text { cxpressāo ce pro:einas co cércoro. }\end{array}$ & 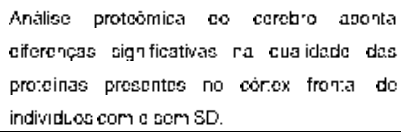 \\
\hline CHOl c: al., $201 ?$. & Ingläs & Revisāo & 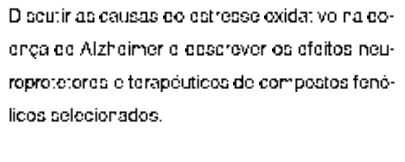 & 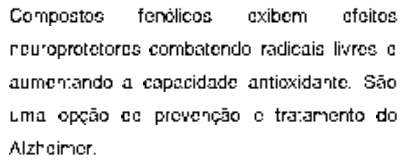 \\
\hline CનOONG ct al., 2015. & Ingläs & Revisāo & 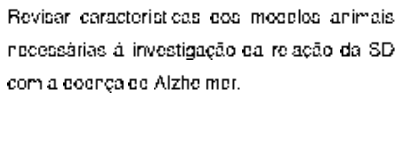 & 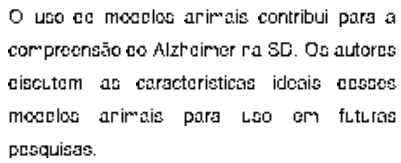 \\
\hline
\end{tabular}


Andreia Araujo Lima Torres

Continua

\begin{tabular}{|c|c|c|c|c|}
\hline DAY et al., 2015 & Inglà̃ & Experimenta & 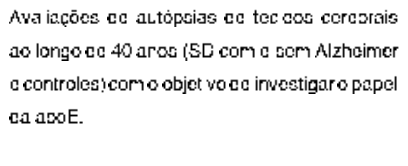 & 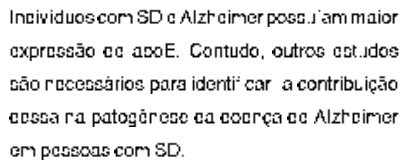 \\
\hline $\begin{array}{l}\text { CE LA TCRRE et al. } \\
2012 .\end{array}$ & Ingläs & Experimente & 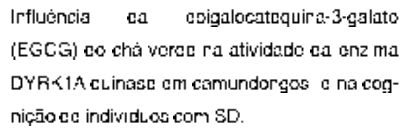 & 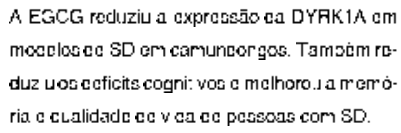 \\
\hline $\begin{array}{l}\text { DI DOMENICC et al. } \\
2013 .\end{array}$ & Ingläs & Experinenta & $\begin{array}{l}\text { Analise' o córtex fro } 7: a \text { ec individeosec ate } 40 \\
\text { aros ec idade. com SD a seus controles neu- } \\
\text { ro:isicos. }\end{array}$ & 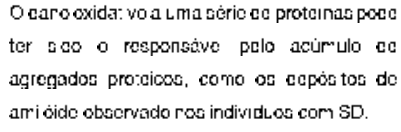 \\
\hline G-EZZO ot al. 2014. & Inglàs & Experimenta & 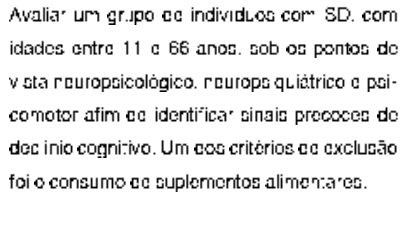 & 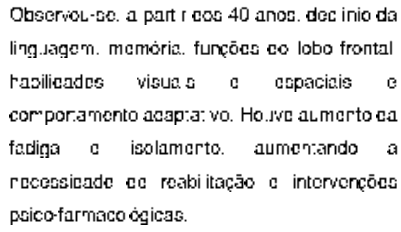 \\
\hline GIL-BE $A$ et al., $201 \underline{\text { }}$. & Ingläs & Expe'inenta & $\begin{array}{l}\text { Es:.Jdar as interaçōes crtre a ccsnervaçāo co- } \\
\text { linérgica c a integridade co ripocampo em mo- } \\
\text { celo ce camuncongo (Tg2576) com cocręa ce } \\
\text { Alzłcimer. }\end{array}$ & 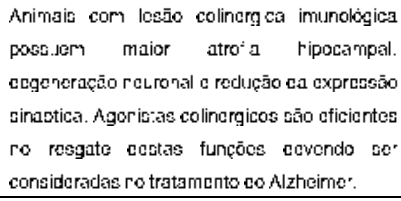 \\
\hline $\begin{array}{l}\text { GRUSZEC } \ll \text { A ct al. } \\
2015 .\end{array}$ & Inglà̃ & Experinenta & $\begin{array}{l}\text { Deserinar o tarranho dos telómeros cm louco- } \\
\text { c tos ce pacieres jovens com SD a gr.jpo con- } \\
\text { trole. }\end{array}$ & 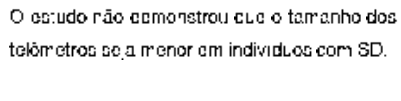 \\
\hline HEAJ ot al., 2012 & Ingliss & คruvisāo & 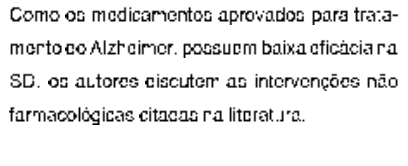 & 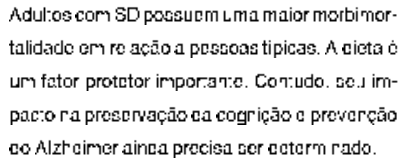 \\
\hline HOAVATH et al., 2015. & Inglàs & Exporinenta & 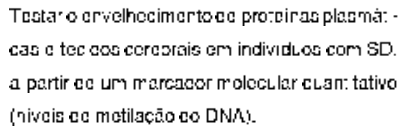 & 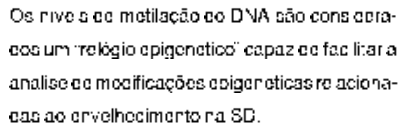 \\
\hline IVER et al., 2012. & Ingläs & Exporinenta & 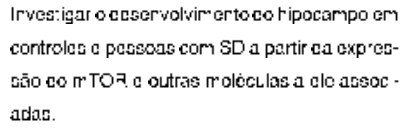 & $\begin{array}{l}\text { Ireividuos com SD abresentam maior ativaçāo } \\
\text { co mTO } \\
\text { precoce para arormalidades ro dacervolvi- } \\
\text { merto a furcionamer:o co ripocampo. }\end{array}$ \\
\hline JEVKIVS et al., 2006 & Inglàs & Experinenta & 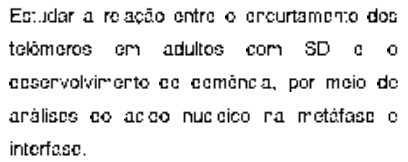 & $\begin{array}{l}\text { O creurtameno cos telómeros foi associado ao } \\
\text { ecservolvirerto ce coerça ce Alzł cimer ra } \\
\text { SC. podendo ser Ltilizado como um marcacor } \\
\text { biolog co para a ceméncia. }\end{array}$ \\
\hline
\end{tabular}


Continua

\begin{tabular}{|c|c|c|c|c|}
\hline JEVKIVS et al., $200 \mathrm{~B}$. & Inglès & Expe'inenta & 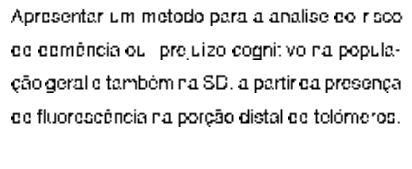 & 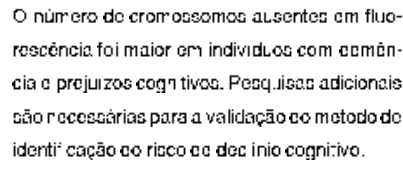 \\
\hline JEVKIVS et al., 2016. & Ingläs & Expe'inente & $\begin{array}{l}\text { Verificar a progressāo das perdas cogn tivas o } \\
\text { furciona s ccvido ao Alztcimer a a sua assoc - } \\
\text { ą̧̄o com o crcurtameq o cos teló méos raSD. }\end{array}$ & 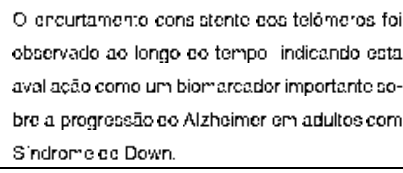 \\
\hline $\begin{array}{l}\text { KARJNA-WEEFA ot al.. } \\
2015 .\end{array}$ & Inglès & Revisāo & 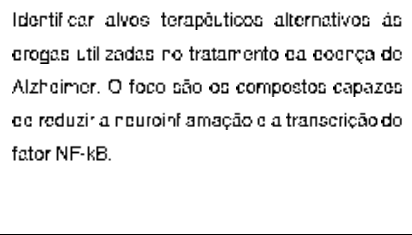 & 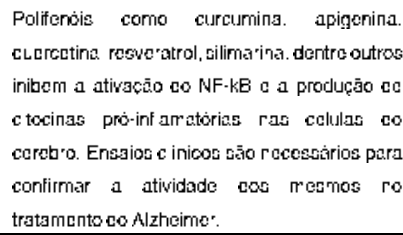 \\
\hline $\begin{array}{l}\text { LIV NGS-OVE et al., } \\
2015\end{array}$ & Inglós & Revisāo & $\begin{array}{l}\text { Avalia a cfetividade ec mecicamentos a suple- } \\
\text { mertos no tra:amerto do dec inio cogni: vo de } \\
\text { pessoas com SD. }\end{array}$ & $\begin{array}{l}\text { Aperas e arigos aterceram aos criterios ce in- } \\
\text { clusâo cos atorcs, impossibilitendo inferéncia } \\
\text { acerca ca cfetividade cos suplementos a cas } \\
\text { crogas invcstigasdos. }\end{array}$ \\
\hline LOPES et al., 2014. & Portlggtès & Revisน̃o & $\begin{array}{l}\text { Combrecnder as peculiar daces co processo de } \\
\text { ervelhecer er idosos com Sindrome ce Down. }\end{array}$ & 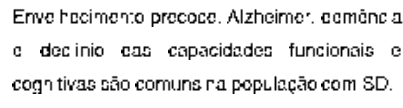 \\
\hline LOTT et al., 2011. & Inglós & Expe'inenta & 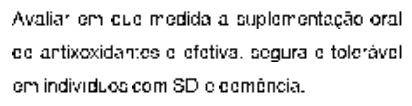 & $\begin{array}{l}\text { Comparaco ao gr.jpo cont ole. os indlividuos su- } \\
\text { plementados rāo asrescrtaram realhorias cog- } \\
\text { nit vas ram cotab lizaçāo co dec inio cogni: vo. }\end{array}$ \\
\hline $\begin{array}{l}\text { NAFQUES: NARREIRO. } \\
2006 .\end{array}$ & Portlugtâs & Revisāo & $\begin{array}{l}\text { Felatar aspectos furcionas co z rco, assim } \\
\text { como a paric saçăo desse ras alteraçôss me- } \\
\text { tabólicas ca SC. }\end{array}$ & 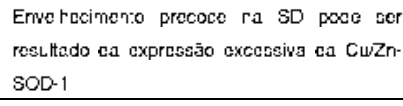 \\
\hline MASSACCESI et al., 2006 & Inglàc & Expe'inenta & 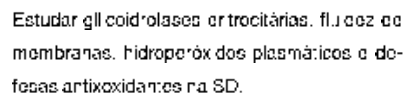 & $\begin{array}{l}\text { O ervelhecimerto cos erit'ce tos na SD ciford } \\
\text { co asresentado en individuos tipicos, com } \\
\text { maior cet'esse oxidativo. }\end{array}$ \\
\hline $\begin{array}{l}\text { MUE-OWA ZI-NANOVA; } \\
\text { DURACKOVA 2012. }\end{array}$ & Inglàs & Revisāo & 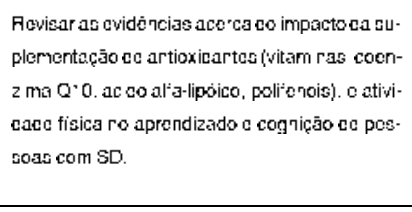 & 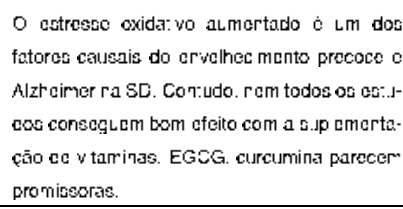 \\
\hline $\begin{array}{l}\text { O3RESH-KOVA et al., } \\
201\end{array}$ & Inglós & Rcvisūo & $\begin{array}{l}\text { Sur" ariar os efcitos protetores cos acidos gra- } \\
\text { xos Ómega-3 a alguns aminoácidos ra preven- } \\
\text { çāo c tra:amerto dos pr reissis tipos cc cemén- } \\
\text { cis. }\end{array}$ & 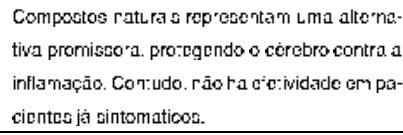 \\
\hline ORMAZAB $A$ L ot al., 2015. & Inglès & Revisนāo & 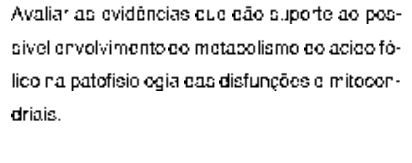 & 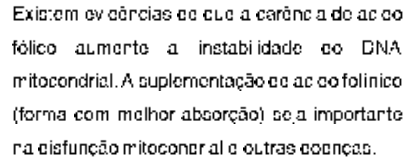 \\
\hline
\end{tabular}


Conclusão

\begin{tabular}{|c|c|c|c|c|}
\hline$P A_{-} A R D C$ et al. 2010. & Ingläs & Revisāo & $\begin{array}{l}\text { Acvisar as cvidéncias ace'ca do crvolvimento } \\
\text { co ce:"esse oxida: vo em várias coerças. inclu- } \\
\text { indo a S ndrore ce Down. }\end{array}$ & 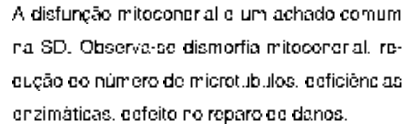 \\
\hline $\begin{array}{l}\text { RISSMAV: DE BLAS: } \\
\text { AFNSTRONG, } 2007 \text {. }\end{array}$ & Inglàs & คevisāo & 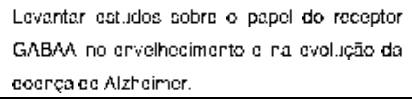 & 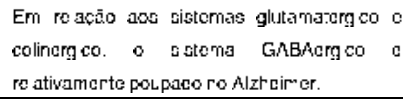 \\
\hline $\begin{array}{l}\text { RISSMAN: MOBLE } \\
2011 .\end{array}$ & Inglès & Rô ธāo & 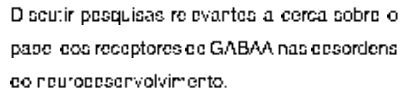 & $\begin{array}{l}\text { O Alzłcimer almerta a inc céncia cc } \\
\text { convu sōes. O fao poce esar re acionado a } \\
\text { alteraçōes roo reccptores GABMA. }\end{array}$ \\
\hline $\begin{array}{l}\text { ROD } \text { नjGUEZ-SUREDA et } \\
\text { al.. } 2015 \text {. }\end{array}$ & Inglès & Expo'inentea & 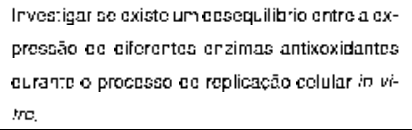 & $\begin{array}{l}\text { F broblastos ec individuos com SD poscuem } \\
\text { maior expressão ce SOD1. o cLe contribui para } \\
\text { ecsectil brio co sistema ce ce cefesa } \\
\text { antioxidante. }\end{array}$ \\
\hline $\begin{array}{l}\text { RUBIO-PEREZ ot al., } \\
2015 .\end{array}$ & Inglès & Expe'inenta & 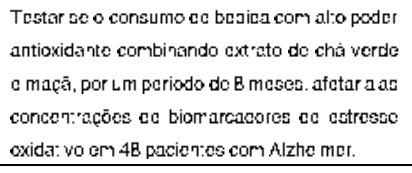 & 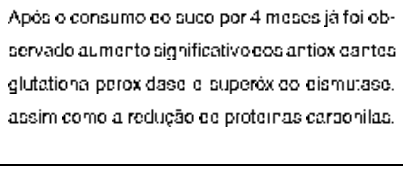 \\
\hline $\begin{array}{l}\text { SPAW: Z नA VG: CHANG. } \\
2015 .\end{array}$ & Inglès & Expe'inenta & 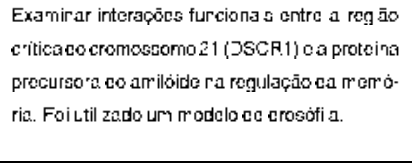 & 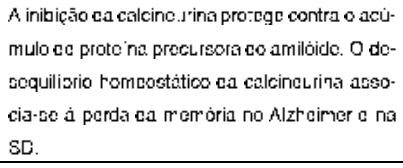 \\
\hline WALEVTI et al., 2010. & Inglès & Expe'inenta & 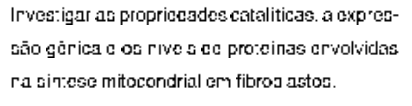 & $\begin{array}{l}\text { A capac cade focforilativa cas mitocóndrias } \\
\text { estí comprometida ra SD. com reduçāo da } \\
\text { ativ dade ca ATP sin:asc. AMPc a PKA. }\end{array}$ \\
\hline VALEVTI et al., 2013. & Inglàs & Expe'inenta & 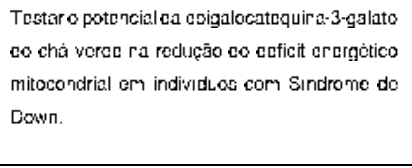 & 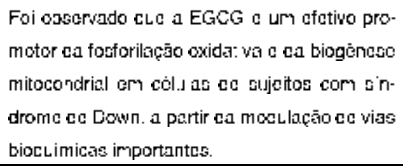 \\
\hline VALEVTI et al., 2016. & Ingläs & Expe'inenta & $\begin{array}{l}\text { Analise ca funçāo mitocordrial em cèlu as co } \\
\text { ripocampo cc rroddo cc SD ce camundorgos } \\
\text { ('s65Dn) com e sem o Leo ce resveratrol. }\end{array}$ & $\begin{array}{l}\text { O resveratrol resta.. ou a biogenese mitocon- } \\
\text { eral a melhoro.J a prol feraçấo cc célu as pro- } \\
\text { genitoras relrais. }\end{array}$ \\
\hline W SEMAN c: al., 2015 & $\mid$ & Rovisสิo & 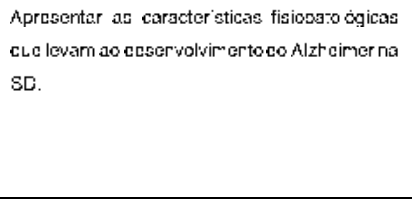 & 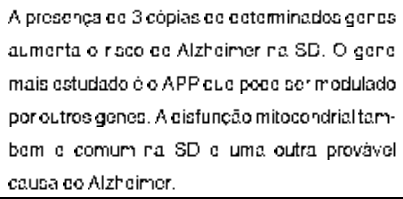 \\
\hline
\end{tabular}

Fonte: dados da pesquisa. 
Os estudos selecionados destacaram diferentes alterações no processo de envelhecimento de indivíduos com SD comparados com a população em geral, incluindo senescência precoce de eritrócitos e do sistema imune (LOPES et al., 2014; MASSACCESI et al., 2006), estresse oxidativo aumentado (MUCHOVÁ; ZITNANOVÁ; DURACKOVÁ, 2014) e disfunção mitocondrial (PALLARDÓ et al., 2010; VALENTI et al., 2016).

Tecnologias modernas permitiram a avaliação de padrões de metilação do DNA. Nesses estudos, foi observado que indivíduos com SD apresentaram uma "assinatura epigenética", que gera maior dificuldade de metilação, o que contribui para o envelhecimento acelerado e o aparecimento precoce de doenças (BACALINI et al., 2015). Tais alterações contribuem para o maior estresse oxidativo.

De fato, as concentrações plasmáticas da enzima antioxidante endógena glutationa (GSH) encontra-se diminuída na SD (AGUILAR-DA-SILVA; MORAES; MORAES, 2003). Já a Cu,Zn-superóxido dismutase -1 (SOD-1), localizada no cromossomo 21, está superexpressa em até $50 \%$ (CASTELLO, 2012) em células como eritrócitos, leucócitos, plaquetas e fibroblastos. Isso proporciona um quadro de agressão endógena constante, dada a acelerada formação de peróxido de hidrogênio $\left(\mathrm{H}_{2} \mathrm{O}_{2}\right)$ e ao desequilíbrio entre a atividade da SOD-1 e glutationa peroxidase (RODRÍGUEZ-SUREDA et al., 2015).

Quando SOD aumenta há uma produção elevada de $\mathrm{H}_{2} \mathrm{O}_{2}$ (Figura 2), que se não for eliminado gera danos ao DNA, fraqueza e atrofia muscular, degeneração de neurônios (MEGUID et al., 2015), envelhecimento precoce, disfunção mitocondrial, modificações bioquímicas e risco aumentado de $\mathrm{Al}$ zheimer (BUTTERFIELD et al., 2014), em idades precoces (DI DOMENICO et al., 2013). O envelhecimento precoce na SD pode ser parcialmente atribuído à hiper expressão de Cu/Zn-SOD1 (MARQUES; MARREIRO, 2006).

Figura 2 - Atuação de enzimas antioxidantes

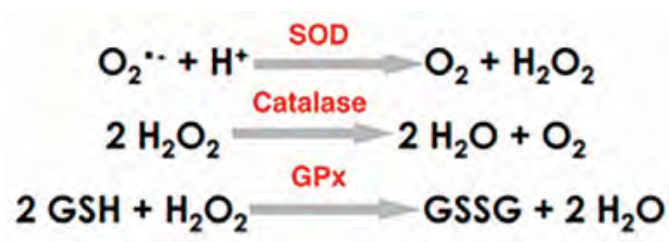

Fonte: Marques e Marreiro (2006).

Glutationa peroxidase (GPx) e catalase (CAT) convertem $\mathrm{H}_{2} \mathrm{O}_{2}$ em água, neutralizando-o. Dessa forma, a deficiência de ferro e selênio é perigosa pois limitará a produção dessas duas importantes enzimas antioxidantes, aumentando o estresse oxidativo.

Pessoas com síndrome de Down possuem um maior risco de desenvolvimento de Alzheimer (CHOONG et al., 2015). Pesquisas nessa área vem aumentando, na tentativa de reduzir as complicações relacionadas à demência, incluindo a mortalidade. Ghezzo et al. (2014) observaram maior declínio cognitivo e fadiga em pessoas com SD após os 40 anos. Um dos critérios de exclusão dos participantes da pesquisa foi o uso de suplementos como vitaminas, minerais, 
ácidos graxos ômega 3 e 6, probióticos, acetilcisteína e ácido lipóico. Tais substâncias apresentam importantes efeitos anti-inflamatórios. Assim, estudos subsequentes precisariam comparar grupos com e sem o uso da suplementação.

Isso porque existem evidências de que o cérebro de indivíduos com doença de Alzheimer é mais inflamado (OBRESHKOVA et al., 2016; GIL-BEA et al., 2012), o que contribui para a perda de funções mentais superiores, alterações progressivas de humor e comportamento, perda da memória, desorientação e dificuldades na fala.

A autópsia do cérebro permite a análise proteômica do tecido. Estudo de Cenini et al. (2015) mostrou diferenças significativas na qualidade das proteínas presentes no córtex frontal de indivíduos com e sem SD, em diferentes faixas etárias. O cérebro de indivíduos com SD e Alzheimer apresentam decréscimo de enzimas importantes para o metabolismo energético cerebral, como alfa-enolase, Fator de elongação Tu (EF-Tu) e da proteína antioxidante peróxido redutase tioredoxina dependente (PRDX3). Existe a expectativa de que esse conhecimento abra futuramente novas possibilidades terapêuticas.

A alta prevalência de Alzheimer nos portadores da síndrome de Down também é atribuída à expressão excessiva de determinados genes localizados no cromossomo 21. Tais genes codificam a produção de proteínas como a precursora amilóide (APP) e a calcineurina, responsável pela fosforilação da proteína Tau (SHAW; ZHANG; CHANG, 2015).
Os custos financeiros, emocionais e familiares em casos de doença de Alzheimer são grandes e aumentam na ausência de uma terapêutica que retarde a progressão dessa doença. Parte das pesquisas focam na neutralização dos radicais livres e na redução da inflamação cerebral com o intuito de se evitar a morte celular e a demência.

Além do papel das enzimas endógenas, estuda-se a necessidade da suplementação de micronutrientes. Apesar de não existirem evidências de que a suplementação de vitaminas e minerais possa retardar o Alzheimer, autores como Mecocci e Polidori (2012) indicam a suplementação de multivitamínicos e minerais no caso de ocorrência de carências nutricionais. Essa recomendação deve-se ao fato de que deficiências de nutrientes dificultam o pleno desenvolvimento, comprometem a imunidade e contribuem para maior estresse oxidativo.

Contudo, Lott et al. (2011) suplementaram, por 2 anos, em estudo aleatorizado, duplo-cego, com grupo controle, $900 \mathrm{UI}$ de alfa-tocoferol, $200 \mathrm{mg}$ de ácido ascórbico, $600 \mathrm{mg}$ de alfa-lipóico a indivíduos com SD e demência (idade média de 50 anos). Os mesmos toleram bem o suplemento e não foram relatados efeitos adversos sérios. Contudo, o tratamento não resultou em melhorias ou estabilização do curso da demência. Os autores discutem que os achados podem ter se dado em função do baixo número de participantes (26 no grupo controle) e do fato da suplementação ter sido iniciada tardiamente (por volta dos 50 anos). Também sugerem que, em 
estudos futuros, as dosagens de cada componente da fórmula seja aumentado. Por fim, referem que as próprias anormalidades cerebrais também podem ter contribuído para a falta de efetividade do tratamento.

De fato, revisão conduzida por Obreshkova et al. (2016), neste momento com o ômega-3, aponta que há efetividade desse na proteção do cérebro contra a inflamação. Contudo, tal efetividade é nula em pessoas com sintomatologias avançadas relacionadas ao Alzheimer, como grandes perdas da memória e desorientação.

Observa-se ainda que indivíduos com síndrome de Down apresentam maior ativação da proteína alvo da rapamicina em mamíferos (mTOR) no cérebro. $\mathrm{O}$ aumento da expressão desta via poderia contribuir para o desenvolvimento de anormalidades precoces no hipocampo (IYER et al., 2014).

Outra hipótese para o Alzheimer é que o estresse oxidativo leva a um aumento da excitação glutamatérgica, a qual contribui para as perdas observadas na doença de Alzheimer (ARUNDINE; TYMIANSKI, 2004). Convulsão, hiperatividade, déficit de atenção, redução da memória e da capacidade de aprendizagem, também sugerem um desequilíbrio entre GABA (ácido gama-aminobutírico), que pode estar diminuído e glutamato, que pode estar aumentado (RISSMAN; MOBLEY, 2011). O próprio envelhecimento também pode danificar receptores para GABA, agravando os sintomas (RISSMAN; DE BLAS; ARMSTRONG, 2007).
Pesquisas mostram que a resistência à insulina e a hiperglicemia também desequilibram a relação GABA/glutamato e aumentam o risco de desenvolvimento de demência. Aproximadamente $90 \%$ dos neurônios do hipocampo são neurotransmissores excitatórios glutamatérgicos enquanto os $10 \%$ restantes são inibitórios, sendo a maioria GABAérgicos. A suplementação de L-teanina, aminoácido do chá verde é um caminho de pesquisa, visto que o composto parece ser eficiente para aumentar os níveis de GABA no cérebro. Rubio-Perez et al. (2015) administraram bebida com extratos de chá verde e maçã para pacientes com Alzheimer por 8 meses. A bebida continha extrato de Camellia sinensis seco com > $98 \%$ de polifenóis, $>80 \%$ de catequinas, $>80 \%$ de epigalocatequina galato e < $40 \%$ cafeína, além de extrato de maçã com $>80 \%$ de polifenóis, expresso em equivalente de catequina. A partir do $4^{\circ}$ mês de uso, observou-se aumento dos antioxidantes glutationa peroxidase e superóxido dismutase, diminuição do estresse oxidativo e redução no aumento de proteínas carboniladas no cérebro.

Em modelos de camundongos com $\mathrm{SD}$, a suplementação de Epigalocatequina-3-galato (EGCG) reduziu ainda a expressão da DYRK1A, enzima cuja superexpressão está associada a déficits cognitivos. A suplementação $(9 \mathrm{mg} / \mathrm{kg} /$ day) também reduziu os déficits cognitivos e melhorou a memória e qualidade de vida de pessoas com SD (DE LA TORRE et al., 2014). A EGCG do chá verde também restaura a capacidade fosforilativa das mitocôndrias, ao aumentar a 
atividade catalítica da ATP sintase e do complexo I e promover a gênese dessas organelas (VALENTI et al., 2013).

A alimentação e a suplementação também podem modular a produção de GABA, já que esta é dependente da disponibilidade de glutamina e vitamina B6. A glutamina é sintetizada no próprio organismo, a partir dos aminoácidos ácido glutâmico, valina e leucina, presentes em carnes, aves, peixes, leguminosas e quinoa (PACÍFICO et al., 2005).

A disfunção mitocondrial observada no Alzheimer é outro fator a aumentar a produção de espécies reativas de oxigênio (CHOI et al., 2012). Mitocôndrias são organelas essenciais para a produção de energia (ATP). Contribuem ainda para a homeostasia do cálcio, plasticidade sináptica, morte celular programada (apoptose) e a liberação de neurotransmissores (ROBERTS et al., 2009).

Quanto maior é a demanda energética de um tecido, mais mitocôndrias ele tem, como é o caso dos músculos, cérebro e trato digestório. Contribuem para a disfunção mitocondrial a formação excessiva de radicais livres, a lipotoxicidade, os fatores genéticos, os processos pró-inflamatórios e a carência de folato (ORMAZABAL et al., 2015), condições que precisam ser observadas.

Polifenóis provenientes de plantas, como curcumina, apigenina, quercetina, resveratrol, silimarina, dentre outros inibem a produção de citocinas pró-inflamatórias nas células do cérebro. Estudos clínicos vêm buscando confirmar a ação desses compostos na prevenção e no tratamento do Alzheimer (KARUNAWEE-
RA et al., 2015). Contudo, não foram encontrados estudos que relatem o uso de tais compostos para a prevenção do Alzheimer e da demência em indivíduos com síndrome de Down.

Uma das hipóteses para o envelhecimento precoce na SD é o encurtamento dos telômeros, complexos DNA-proteína localizados nas extremidades dos cromossomos (JENKINS et al., 2006, 2008). Contudo, em estudo publicado por Gruszecka et al. (2015), o tamanho também foi maior em pacientes com síndrome de Down do que no grupo controle (sem SD). Já Jenkins et al. (2016) observou encurtamentos de telômeros consistentes em indivíduos com SD.

Por fim, as alterações da microbiota intestinal durante o envelhecimento também foram objeto de avaliação. $\mathrm{Na}$ $\mathrm{SD}$, parece haver maior colonização por bactérias patogênicas Parasporobacterium e Sutterella, com redução de Veillonellaceae (BIAGI et al., 2014).

\section{Discussão}

Fatores genéticos resultam no aumento da frequência de doença de Alzheimer em pessoas com Síndrome de Down. Apesar das semelhanças entre o Alzheimer de idosos neurotípicos e a demência na SD, o tratamento medicamentoso é pouco efetivo nos últimos. Dessa forma, novas abordagens são necessárias (BALLARD et al., 2016). Ainda existem poucos estudos experimentais controlados e desenhados de forma adequada nesta área (LIVINGSTONE et al., 2015). Assim, novas pesquisas 
que avaliem estratégias nutricionais potencialmente capazes de amenizar os sintomas relacionados ao Alzheimer e à prevenção da demência relacionadas à SD fazem-se necessárias.

No apanhado apresentado foi possível visualizar o início da construção deste conhecimento. Pesquisas com indivíduos típicos (sem síndrome de Down) mostram, por exemplo, que a suplementação de GABA pode melhorar a qualidade do sono (YAMATSU et al., 2015), reduzir a glicação da albumina (LI et al., 2015), sintomas da ansiedade (ABDOU et al., 2006) e depressão (OKADA et al., 2000) em idosos. Infere-se que a administração de GABA via oral possa amenizar os mesmos problemas na Síndrome de Down. Dessa forma, sugerem-se estudos clínicos com esse grupo de indivíduos.

Uma das possíveis causas para a maior prevalência de Alzheimer e demência neste grupo é o estresse oxidativo. Além dos mecanismos antioxidantes endógenos, polifenóis antioxidantes são também efetivos em neutralizar o peróxido de hidrogênio. Sucos de frutas e verduras ricos em polifenóis parecem uma boa alternativa para retardar a progressão do Alzheimer (DAI et al., 2006) em indivíduos típicos. Um exemplo é o suco de romã (BOOKHEIMER et al., 2013), que reduz o dano oxidativo inibe citocinas inflamatórias, aumenta a produção de energia (AKBAR et al., 2015) e parece melhorar memória, aprendizagem, função motora e reduzir a ansiedade (SUBASH et al., 2015).

Polifenóis presentes no chá verde, principalmente a epigalocatequina-3- -galato (EGCG) reduz a atividade da DYRK1A. O gene para essa enzima está presente no cromossomo 21 e sua atividade aumentada está associada ao maior risco de Alzheimer (MUCHOVÁ; ZITNANOVÁ; DURACKOVÁ, 2014). Os efeitos do EGCG puro foi comparado com a administração do suplemento em diferentes concentrações em relação à melhoria óssea em ratos modelos com SD. O EGCG puro teve menor feito do que em concentrações de 50 a $90 \%$. Uma possível explicação é a de que outros polifenóis presentes no extrato podem ter um papel na disponibilidade e manutenção da ação do EGCG (ABEYSEKERA et al., 2016). Assim, não seria recomendada a suplementação isolada de EGCG, e, sim, do extrato de Camellia sinensis, por sua composição, ou mesmo de infusões feitas com a própria planta, administradas puras ou misturadas a sucos, como no estudo de Rubio-Perez et al. (2015).

Polifenóis das uvas roxas, como o resveratrol, também vem sendo estudados. Existem evidências de que esses atenuam a neuropatia e o declínio cognitivo, além de reduzir a formação de placas beta amilóides no cérebro (HO et al., 2010; NICHOLS, 2014; PORAT; ABRAMOWITZ; GAZIT, 2006). Contudo, dosagens na síndrome de Down precisam ser investigadas. Estudo de Valenti et al. (2016), em modelos de SD em camundongos, mostrou que a bioenergética mitocondrial esteve comprometida, mas não associada ao acúmulo de radicais livres. Ou seja, a disfunção mitocondrial parece ser inerente à SD e não necessariamente resultado do estresse oxidativo. 
A suplementação de resveratrol serviu para restar justamente a biogênese mitocondrial e melhorar a proliferação de células progenitoras neurais.

Já Masci et al. (2015) mostraram em estudo in vitro que sucos de brássicas protegem as células contra a toxicidade do peptídio beta amilóide. O suco também aumentou os níveis de glutationa e os níveis de RNAm para enzimas antioxidantes. A expectativa é de que a suplementação de polifenóis por períodos prolongados reduza o risco de demência também em pessoas com SD e Alzheimer, o que ainda precisa ser estudado.

Por fim, compostos ativos da cúrcuma parecem promissores no tratamento do Alzheimer (HAMAGUCHI; ONO; YAMADA, 2010). Tais compostos interagem com proteínas, enzimas e metais e receptores no organismo, inibindo a produção de substancias inflamatórias locais (FRAUTSCHY et al., 2002) e a fosforilação da proteína Tau (MA et al., 2009). Contudo, a curcumina não é muito bem absorvida. Dessa forma, no caso de suplementação deve-se aliá-la à piperina. No alimento, a cúrcuma pode ser associada à pimenta vermelha, a óleos ou à quercetina (presente em cebolas, maçãs, brócolis, espinafre, couve e chicória) com o intuito de melhorar sua disponibilidade. A curcumina também é sensível ao calor. Por isso, o açafrão deve ser adicionado apenas ao final das preparações.

No sentido de minimizar a ativação do mTOR, sugere-se a redução do consumo de alimentos com alto índice glicêmico e de proteínas de origem ani- mal (carnes, ovos e laticínios). Esses alimentos contribuem para a ativação do mTOR, aumentando o risco de Alzheimer e outras doenças (MELNIK; JOHN; PLEWIG, 2013).

Uma das hipóteses para o envelhecimento é a redução no tamanho dos telômeros (JENKINS et al., 2016). Contudo, no estudo de Gruszecka et al. (2015) não foi possível demonstrar diferenças significativas entre os grupos com e sem SD. Dessa forma, outros estudos são indicados para que os telômeros possam ser utilizados com confiança como um indicador da velocidade de envelhecimento na SD ou indicador do risco de demência. É importante ter em mente que o tamanho do tamanho do telômero varia com outros fatores além da genética, incluindo idade, sexo, perfil hormonal, condições relacionadas ao estilo de vida, alimentação e composição corporal.

Existem relatos que o folato, por exemplo, é uma das vitaminas que influenciam o tamanho dos telômeros (FENECH, 2012). Porém, não foram encontrados estudos experimentais durante esta revisão, o que não descaracteriza o papel da vitamina na saúde de indivíduos com SD. Coppède (2015) realizou revisão sistemática que mostrou uma associação positiva entre a carência de vitamina B9 na gestação e o aumento das chances de ocorrência de SD no concepto. Contudo, há a necessidade de desenhos experimentais com maiores números de indivíduos, para que haja mais clareza acerca da contribuição materna nas modificações epigenéticas observadas. 
Outro marcador que começa a ser estudado para avaliação do envelhecimento é o "relógio epigenético", o qual se baseia na determinação da idade do DNA mitocondrial (DNAm). O envelhecimento do DNAm correlaciona-se de forma forte e positiva com o envelhecimento cronológico em indivíduos neurotípicos. $\mathrm{Na}$ $\mathrm{SD}$, o envelhecimento do DNAm parece ser ainda mais acelerado (HORVATH et al., 2015).
Apesar da limitação no número de pesquisas abordados, algumas recomendações de suplementos podem ser feitas, a partir dos estudos analisados. As fórmulas sugeridas precisam ser adaptadas a cada caso, sob orientação de um nutricionista capacitado na área (Tabela 4):

Tabela 4 - Sugestão de fórmulas para adultos com síndrome de Down

\begin{tabular}{|c|c|c|c|}
\hline \multicolumn{2}{|c|}{ Fórmula antioxidante (opção 1) } & \multicolumn{2}{|c|}{ Fórmula antioxidante (opção 2) } \\
\hline EGCG & $100 \mathrm{mg}$ & Resveratrol & $25 \mathrm{mg}$ \\
\hline Curcumina & $100 \mathrm{mg}$ & EGCG & $100 \mathrm{mg}$ \\
\hline Piperina - $5 \mathrm{mg}$ & $5 \mathrm{mg}$ & Excipiente qsp & 1 cápsula \\
\hline Excipiente qsp & 1 cápsula & Mande 60 unidades & \\
\hline \multicolumn{2}{|c|}{$\begin{array}{l}\text { Posologia: Tomar } 1 \text { cápsula, } 2 \text { vezes ao dia. } \\
\text { Fórmula para equilíbrio da microbiota }\end{array}$} & \multicolumn{2}{|c|}{$\begin{array}{l}\text { Posologia: Tomar } 1 \text { cápsula, } 2 \text { vezes ao dia. } \\
\text { Ativo para a produção de GABA }\end{array}$} \\
\hline L. acidophilus - 450 milhões de UFC & & L-theanina & $100 \mathrm{mg} / \mathrm{dia}$ \\
\hline L. helveticus - 190 milhões de UFC & & Excipiente qsp & 1 cápsula \\
\hline L. plantarum - 100 milhões de UFC & & Posologia: Tomar 1 dose, 2 vezes ao dia & \\
\hline B. lactis - 75 milhões de UFC & & Suporte para metilação & \\
\hline B. longum - 110 milhões de UFC & & Vitamina B6 & $250 \mathrm{mg}$ \\
\hline B. brevis - 75 milhões de UFC & & Vitamina B9 (ácido fólico) & $10 \mathrm{mg}$ \\
\hline FOS - $500 \mathrm{mg}$ & & Vitamina B12 & $5 \mathrm{mg}$ \\
\hline \multicolumn{2}{|c|}{ Excipiente qsp - 1 dose (cápsulas ou sachê) } & Excipiente qsp & Cápsulas \\
\hline \multicolumn{2}{|c|}{ Posologia: Tomar 1 dose ao dia, antes de dormir } & Posologia: Tomar 1 dose, à noite & \\
\hline
\end{tabular}

Fonte: dados da pesquisa.

\section{Conclusões}

Esta revisão abordou estudos que relacionassem a alimentação e a suplementação de compostos específicos ao processo de envelhecimento em indivíduos com SD. A literatura científica reconhece o envelhecimento prematuro do cérebro como uma característica comum da SD. Nos artigos selecionados nesta revisão, os autores apontam preocupação com $o$ desenvolvimento da doença de Alzheimer acompanhada de demência. A maior prevalência do problema em pessoas com SD parece dever-se às características genéticas próprias desses indivíduos, associadas a maior estresse oxidativo e disfunção mitocondrial.

Apesar de a resistência à insulina e hiperglicemia aumentarem o risco de 
demência, o tratamento dietético, incluindo dietas com baixo índice glicêmico ou outras estratégias para a redução da glicemia não foi abordado nos estudos investigados. Dessa forma, pesquisas que avaliem diferentes composições dietéticas na evolução do Alzheimer e demência ainda fazem-se necessárias.

A suplementação de nutrientes pode ser uma aliada, especialmente no caso de dietas monótonas ou insuficientes. Destaca-se, entretanto, que super dosagens não são recomendas devido ao risco de comprometimento do metabolismo de neurotransmissores, depleção de grupamentos metil e neurotoxicidade (ZHOU et al., 2013). Como inovação, este trabalho trouxe algumas sugestões de fórmulas, em dosagens adequadas para adultos, apoiadas nas pesquisas elencadas. Ainda assim, tais fórmulas devem ser analisadas caso a caso, por um nutricionista capacitado na área.

Destaca-se a necessidade de estudos adicionais na área, uma vez que existem poucos biomarcadores para o envelhecimento, o que dificulta a compreensão acerca dos fatores relacionados ao envelhecimento na SD. Ainda se discute se a síndrome é responsável pelo envelhecimento acelerado ou se há um aumento da vulnerabilidade à condições tipicamente associadas ao envelhecimento, como o próprio Alzheimer. Futuros estudos, especialmente epigenéticos, poderão contribuir ao avaliar a extensão na qual o envelhecimento precoce do DNA, especialmente do mitocondrial, correlaciona-se à velocidade de aparecimento de doenças. Espera-se ainda que estudos na área de nutrigenética e nutrigenômica contribuam para o aprofundamento do conhecimento acerca da efetividade da suplementação de nutrientes e outros compostos bioativos na desaceleração do envelhecimento.

Uma das limitações desta pesquisa refere-se ao fato de um único avaliador ter procedido à análise dos artigos científicos selecionados para a revisão. Outra limitação foi a opção metodológica, a qual permitiu a inclusão de estudos de revisão no trabalho. Por fim, apenas duas bases de dados foram utilizadas neste trabalho (Scielo e Bireme), havendo a necessidade de expansão da revisão para melhor compreensão da temática.

\section{Nutritional aspects associated with aging of people with Down Syndrome: integrative review}

\section{Abstract}

This paper presents an integrative review on the latest research involving nutrition and supplementation during the aging of individuals with Down syndrome. Due to the high incidence of Alzheimer's and dementia it is relevant to study the contributing factors leading to a better quality of life in this population. The financial, emotional and family costs of Alzheimer's disease are large and increase in the absence of a treatment that slows its progression and complications. Thus, we conducted a literature search, encompassing the last 10 years, in Scielo and Bireme databases. The objective was to study how diet and supplementation of specific compounds relate to the aging process in individuals with DS. The analysis of the extracted do- 
cuments led to the reading of 38 papers. Part of the research in the area of nutrition focused on the importance of neutralizing free radicals and reducing brain inflammation as remediative strategies targeting the most common complications encountered in this population. Other aspects related to the aging of individuals with Down syndrome were addressed.

Keywords: Down syndrome, Alzheimer Disease, Child Nutrition, Supplementary Feeding

\section{Referências}

ABEYSEKERA, I. et al. Differential effects of Epigallocatechin-3-gallate containing supplements on correcting skeletal defects in a Down syndrome mouse model. Molecular Nutrition \& Food Research, v. 60, n. 4, p. 717-726, 2016.

ABDOU, A. M. et al. Relaxation and immunity enhancement effects of $\square$-Aminobutyric acid (GABA) administration in humans. BioFactors, v. 26, n. 3, p. 201-208, 2006.

AGUILAR-DA-SILVA, R. H.; MORAES, T. P.; MORAES, G. Implicações do estresse oxidativo sobre o metabolismo eritrocitário de pessoas com Síndrome de Down. Revista Brasileira de Hematologia e Hemoterapia, v. 25, n. 4, p. 231-237, 2003.

ARUNDINE, M.; TYMIANSKI, M. Molecular mechanisms of glutamate-dependent neurodegeneration in ischemia and traumatic brain injury. Cellular and molecular life sciences, v. 61, n. 6, p. 657-668, 2004.

BACALINI, M. G. et al. Identification of a DNA methylation signature in blood cells from persons with Down Syndrome. Aging, v. 7, n. 2, p. 82-93, 2015.

BALLARD, C. et al. Dementia in Down's Syndrome. The Lancet Neurology, v. 15, n. 6, p. $622-636,2016$.
BIAGI, E. et al. Gut Microbiome in Down Syndrome. PLoS One, v. 9, n. 11, e112023, 2014.

BOOKHEIMER, S. Y. et al. Pomegranate Juice Augments Memory and fMRI Activity in Middle-Aged and Older Adults with Mild Memory Complaints. Evidence-Based Complementary and Alternative Medicine, ID 946298, 14p., 2013.

BUTTERFIELD, D. A. et al. Redox proteomics analysis to decipher the neurobiology of Alzheimer-like neurodegeneration: overlaps in Down's syndrome and Alzheimer's disease brain. The Biochemical Journal, v. 463, n. 2, p. 177-189, 2014.

CENINI, G. et al. An Investigation of the Molecular Mechanisms Engaged Prior and Subsequent to the Development of Alzheimer Disease Neuropathology in Down Syndrome: A Proteomics Approach. Free Radical Biology $\&$ Medicine, Nov. 2015 In Press.

CHOI, D. Y. et al. Antioxidant properties of natural polyphenols and their therapeutic potentials for Alzheimer's disease. Brain Research Bulletin, v. 87, n. 2-3, p. 144-153, 2012.

CHOONG, X. Y. et al. Dissecting Alzheimer disease in Down syndrome using mouse models. Frontiers in Behavioral Neuroscience, v. 9, 2015.

COPPEDÈ, F. et al. DNMT3B promoter polymorphisms and maternal risk of birth of a child with Down syndrome. Human Reproduction, v. 28, n. 2, p. 545-550, 2013.

DAI, Q. et al. Fruit and Vegetable Juices and Alzheimer's Disease: The Kame Project. The American Journal of Medicine, v. 119, n. 9, p. 751-759, 2006.

DE LA TORRE, R. et al. Epigallocatechin3-gallate, a DYRK1A inhibitor, rescues cognitive deficits in Down syndrome mouse models and in humans. Molecular Nutrition \& Food Research, v. 58, n. 2, p. 278-288, 2014. 
DELCURTO, H.; WU, G.; SATTERFIELD, M. C. Nutrition and reproduction: links to epigenetics and metabolic syndrome in offspring. Current Opinion in Clinical Nutrition \& Metabolic Care, v. 16, n. 4, p. 385-391, 2013.

DI DOMENICO, F. et al. Impairment of proteostasis network in Down syndrome prior to the development of Alzheimer's disease neuropathology: Redox proteomics analysis of human brain. Biochimica et Biophysica Acta (BBA) - Molecular Basis of Disease, v. 1832, n. 8, p. 1249-1259, 2013.

DOMINGUEZ-SALAS, P. et al. Maternal nutrition at conception modulates DNA methylation of human metastable epialleles. Nature Communications, v. 5, n. 3.746, 7p., 2014.

FENECH, M. Folate (vitamin B9) and vitamin B12 and their function in the maintenance of nuclear and mitochondrial genome integrity. Mutation Research / Fundamental and Molecular Mechanisms of Mutagenesis, v. 733, n. 1-2, p. 21-33, 2012.

FRAUTSCHY, S. A. et al. Phenolic anti-inflammatory antioxidant reversal of Abeta-induced cognitive deficits and neuropathology. Neurobiololgy of Aging, v. 22, p. 993-1005, 2002.

GHEZZO, A. et al. Age-related changes of adaptive and neuropsychological features in persons with Down Syndrome. PLoS ONE, v. 24, n. 9, e113111, 2014.

GIL-BEA, F.J. et al. Cholinergic denervation exacerbates amyloid pathology and induces hippocampal atrophy in Tg2576 mice. Neurobiology of Disease, v. 48, p. 439-446, 2012.

GRUSZECKA, A. et al. Telomere Shortening in Down Syndrome Patients - When Does It Start? DNA and Cell Biology, v. 34, n. 6, p. 412-417, 2015.

HAMAGUCHI, T.; ONO, K.; YAMADA, M. Review: Curcumin and Alzheimer's Disease. CNS Neuroscience \& Therapeutics, v. 16, p. 285-297, 2010.
HEAD, E. et al. Aging and Down Syndrome. Current Gerontology and Geriatrics Research, v. 2012, ID 412536, 6p., 2012.

HO, L. et al. Heterogeneity in Red Wine Polyphenolic Contents Differentially Influences Alzheimer's Disease-type Neuropathology and Cognitive Deterioration. Journal of Alzheimer's Disease, v. 16, n. 1, p. 59-72, 2010.

HORVATH, S. et al. Accelerated epigenetic aging in Down syndrome. Aging Cell, v. 14, n. 3, p. 491-495, 2015.

IYER, A. M. et al. mTOR Hyperactivation in Down Syndrome Hippocampus Appears Early During Development. Journal of Neuropathology \& Experimental Neurology, v. 73, n. 7, p. 671-683, 2014.

JENKINS, E. C. et al. Increased "absence" of telomeres may indicate Alzheimer's disease/ dementia status in older individuals with Down syndrome. Neuroscience Letters, v. 440, p. 340-343, 2008.

JENKINS, E. C. et al. Telomere longitudinal shortening as a biomarker for dementia status of adults with Down syndrome. American Journal of Medical Genetics, v. 171B, v. 2, p. 169-174, 2016.

JENKINS, E. C. et al. Telomere shortening in T lymphocytes of older individuals with Down syndrome and dementia. Neurobiology of Aging, v. 27, n. 7, p. 941-945, 2006.

KARUNAWEERA, N. et al. Plant polyphenols as inhibitors of NF- $\kappa \mathrm{B}$ induced cytokine production - a potential anti-inflammatory treatment for Alzheimer's disease? Frontiers in Molecule Neuroscience, v. 8, n. 24, 2015.

KIRWAN, P. et al. Development and function of human cerebral cortex networks from pluripotent stem cells. Development, v. 142 , n. 18, p. 3178-3187, 2015.

LI, J. et al. Study of GABA in Healthy Volunteers: Pharmacokinetics and Pharmacodynamics. Frontiers in Pharmcology, v. 6, art. 260, 9p., 2015.

RBCEH, Passo Fundo, v. I3, n. 3, p. 367-387, set./dez. 2016 
LIVINGSTONE, N. et al. Pharmacological interventions for cognitive decline in people with Down syndrome. Cochrane Database of Systematic Reviews, n. 10, 2015. DOI: 10.1002/14651858.CD011546.pub2.

LOPES, B. S. et al. A Síndrome de Down e o processo de envelhecer: revisão sistemática. Revista Kairós Gerontologia, v. 17, n. 4, p. 141-155, 2014.

LOTT, I. T. et al. Down syndrome and dementia: A randomized, controlled trial of antioxidant supplementation. American Journal of Medical Genetics, v. 155, n. 8, p. 1939-1948, 2011.

LU, J. et al. Global hypermethylation in fetal cortex of Down syndrome due to DNMT3L overexpression. Human Molecular Genetics, in press, 2016.

MA, Q. L. et al. Beta-Amyloid Oligomers Induce Phosphorylation of Tau and Inactivation of Insulin Receptor Substrate via c-Jun N-Terminal Kinase Signaling: Suppression by Omega-3 Fatty Acids and Curcumin. Journal of Neuroscience, v. 29, n. 28, p. 9078-9089, 2009 .

MARQUES, R. C.; MARREIRO, D. N. Aspectos metabólicos e funcionais do zinco na síndrome de Down. Revista de Nutrição, Campinas, v. 19, n. 4, p. 501-510, ago. 2006.

MASCI, A. et al. Neuroprotective Effect of Brassica oleracea Sprouts Crude Juice in a Cellular Model of Alzheimer's Disease. Oxidative Medicine and Cellular Longevity, ID 781938, 17 p., 2015.

MASSACCESI, L. et al. Erythrocyte glycohydrolases in subjects with trisomy 21: could Down's Syndrome be a model of accelerated ageing? Mechanisms of Ageing and Development, v. 127, n. 4, p. 324-331, 2006.

MATHUSWAMY, S.; AGARWAL, S. Do the MTHFR gene polymorphism and Down syndrome pregnancy association stands true? A case-control study of Indian population and meta-analysis. Egyptian Journal of Medican Human Genetics, v. 17, n. 1, p. 87-97, 2016.
MECOCCI, P.; POLIDORI, M. C. Antioxidant clinical trials in mild cognitive impairment and Alzheimer's disease. Biochemica et Biophysica Acta, v. 1822, n. 5, p. 631-638, 2012.

MEGUID, N. A. et al. Antioxidant activity in Egyptian children with Down syndrome before and after nutritional supplementation. Journal of Chemical and Pharmaceutical Research, v. 7, n. 2, p. 324-331, 2015.

MELNIK, B. C.; JOHN, S. M.; PLEWIG, G. Acne: Risk Indicator for Increased Body Mass Index and Insulin Resistance. Acta Dermato-Venereologica, v. 93, p. 644-649, 2013.

MENTIS, A. F. Epigenomic engineering for Down syndrome. Neuroscience \& Biobehavioral Reviews, in press, 2016.

MUCHOVÁ, J.; ZITNANOVÁ, I.; DURACKOVÁ, Z. Oxidative Stress and Down Syndrome. Do Antioxidants Play a Role in Therapy? Physiological Research, v. 63, p. 535-542, 2014.

NICHOLS, W. N. Hyperphosphorylation of Tau Protein in Down's Dementia and Alzheimer's Disease: Methylation and Implications in Prevention and Therapy. Alzheimer's Disease \& Parkinsonism, v. 4, n. 5, 8p., 2014.

OBRESHKOVA, D. P. et al. Ômega-3 polyunsaturated fatty acids and some amino acids in early prevention of Alzheimer's disease. World Journal of Pharmacy and Pharmaceutical Sciences, v. 5, n. 9, p. 2379-2391, 2016. OKADA, T. et al. Effect of the defatted rice germ enriched with GABA for sleeplessness, depression, autonomic disorder by oral administration. J. Japanese Society Food Sci. Techn, v. 47, n. 8, p. 596-603, 2000.

ORMAZABAL, A. et al. Can folic acid have a role in mitochondrial disorders? Drug Discovery Today, v. 20, n. 11, p. 1349-1354, 2015. PACÍFICO, S. L.; LEITE, H. P.; CARVALHO, W. B. A suplementação de glutamina é benéfica em crianças com doenças graves? 
Revista de Nutrição, Campinas, v. 18, n. 1, p. 95-104, 2005.

PALLARDÓ, F. V. et al. Mitochondrial dysfunction in some oxidative stress-related genetic diseases: Ataxia-Telangiectasia, Down Syndrome, Fanconi Anaemia and Werner Syndrome. Biogerontology, v. 11, p. 401-419, 2010.

PARKER, S. E. et al. Updated National Birth Prevalence estimates for selected birth defects in the United States, 2004-2006. Birth Defects Research, Part A, Clinical and molecular teratology, v. 88 , n. 12 , p. 1008 1016, 2010.

PORAT, Y.; ABRAMOWITZ, A.; GAZIT, E. Inhibition of amyloid fibril formation by polyphenols: structural similarity and aromatic interactions as a common inhibition mechanism. Chemical Biology \& Drug Design, v. 67. n. 1, p. 27-37, 2006.

RISSMAN, R. A.; DE BLAS, A. L.; ARMSTRONG, D. M. GABA(A) receptors in aging and Alzheimer's disease. Journal of Neurochemistry, v. 103, n. 4, p. 1285-1295, 2007.

RISSMAN, R. A.; MOBLEY, W.C. Implications for treatment: GABAa receptors in aging, Down syndrome and Alzheimer's disease. Journal of Neurochemistry, v. 117, n. 4, p. 613-622, 2011.

ROBERTS, R. A. et al. Nitrative and oxidative stress in toxicology and disease. Toxicological sciences: an Official Journal of the Society of Toxicology, v. 112, n. 4, p. 16-19, 2009.

RODRÍGUEZ-SUREDA, V. et al. Intracellular Oxidant Activity, Antioxidant Enzyme Defense System, and Cell Senescence in Fibroblasts with Trisomy 21. Oxidative Medicine and Cellular Longevity, 509241, 2015. RUBIO-PEREZ, J. M. et al. Effects of an antioxidant beverage on biomarkers of oxidative stress in Alzheimer's patients. European Journal of Nutrition, v. 55, n. 6, p. 2105-2116, 2015.
SHAW, J. L.; ZHANG, S.; CHANG, K. T. Bidirectional Regulation of Amyloid Precursor Protein-Induced Memory Defects by Nebula/DSCR1: A Protein Upregulated in Alzheimer's Disease and Down Syndrome. Journal of Neuroscience: the Official Journal of the Society of Neuroscience, v. 35, n. 32, p. 11374-11383, 2015.

SILVA, M. et al. Carcinoma gástrico e gastrite crónica: regulação epigenéticaica por metilação dos genes CDH1 (Caderina-E), CDKN2A (p16INK4A), PTGS2 (COX2) e EGFR. Acta Medica Portuguesa, v. 23, p. 5-14, 2010.

STAGNI, F. et al. Timing of therapies for Down syndrome: the sooner, the better. Frontiers in Behavioral Neuroscience, v. 6, n. 9, p. 265, 2015.

STRUPP, B. J. et al. Maternal Choline Supplementation: A Potential Prenatal Treatment for Down Syndrome and Alzheimer's Disease. Current Alzheimer Research, v. 13, n. 1, p. 97-106, 2016.

SUBASH, S. et al. Long-term (15 mo) dietary supplementation with pomegranates from Oman attenuates cognitive and behavioral deficits in a transgenic mice model of Alzheimer's disease. Nutrition, v. 31, n. 1, p. 223-229, 2015.

THIEL, R.; FOWKES, S. W. Down syndrome and thyroid dysfunction: should nutritional support be the first-line treatment? Medical hypotheses, v. 69, n. 4, p. 809-815, 2007.

VALENTI, D. et al. Impairment of F1F0-ATPase, adenine nucleotide translocator and adenylate kinase causes mitochondrial energy deficit in human skin fibroblasts with chromosome 21 trisomy. Biochemical Journal, v. 431, n. 2, p. 299-310, 2010.

VALENTI, D. et al. Epigallocatechin-3-gallate prevents oxidative phosphorylation deficit and promotes mitochondrial biogenesis in human cells from subjects with Down's syndrome. Biochimica et Biophysica Acta, v. 1832 , n. 4 , p. 542-552, 2013. 
VALENTI, D. et al. The polyphenols resveratrol and epigallocatechin-3-gallate restore the severe impairment of mitochondria in hippocampal progenitor cells from a Down syndrome mouse model. Biochemica et Biophysica Acta, v. 1862, n. 6, p. 1093-1104, 2016.

WESSELS, I. Epigenetica and Mental Deficiencies. Current Nutrition Reports, v. 3, n. 3, p. 196-203, 2014.

WISEMAN, F. K. et al. A genetic cause of Alzheimer disease: mechanistic insights from Down syndrome. Nature Reviews Neuroscience, v. 16, p. 564-567, 2015.

WU, J. H.; MORRIS, J. K. Trends in maternal age distribution and the live birth prevalence of Down's syndrome in England and Wales: 1938-2010. European Journal of Human Genetics, v. 21, p. 943-947, 2013a.

WU, J. H.; MORRIS, J. K. The population prevalence of Down's syndrome in England and Wales in 2011. European Journal of Human Genetics, v. 21, p. 1016-1019, 2013b. YAMATSU, A. et al. The Improvement of Sleep by Oral Intake of GABA and Apocynum venetum Leaf Extract. Journal of Nutritional Science and Vitaminology, v.61, n. 2, p. 182-187, 2015.

ZHOU, S. S. et al. Early Infant Exposure to Excess Multivitamin: A Risk Factor for Autism? Autism Research and Treatment, ID 963697, 8p., 2013.

ZIGMAN, W. B. Atypical aging in Down Syndrome. Developmental Disabilities Research Reviews, v. 18, n. 1, p. 51-67, 2013. 